\title{
Fully automated data collection and remote access in macromolecular crystallography beamlines at the Photon Factory, Japan
}

\author{
Y. Yamada ${ }^{1,2}$, A. Shinoda ${ }^{1}$, N. Matsugaki ${ }^{1,2}$, M. Hikita ${ }^{1,2}$, M. Hiraki ${ }^{2,3}$, T. Senda ${ }^{1,2}$ \\ ${ }^{1}$ Structural Biology Research Center, Institute of Materials Structure Science, High Energy Accelerator Research Organization, 1-1 \\ Oho, Tsukuba, Ibaraki, Japan, ${ }^{2}$ School of High Energy Accelerator Science, The Graduate University for Advanced studies, 1-1 Oho, \\ Tsukuba, Ibaraki, Japan, ${ }^{3}$ Mechanical Engineering Center, Applied Research Laboratory, High Energy Accelerator Research \\ Organization, 1-1 Oho, Tsukuba, Ibaraki, Japan,
} yusuke.yamada@kek.jp

Structural Biology Research Center in the Photon Factory (PF), Japan, has five macromolecular crystallography (MX) beamlines at two synchrotron radiation rings, PF and PF-AR. The end stations of all the beamlines are equipped with sample exchange robots [1], highprecision diffractometers, and pixel array detectors, which are controlled by a common control software. This enables us to realize a fully automated and unattended data collection and a remote interactive data collection at all beamlines.

For a fully automated data collection, we developed a dedicated software SIROCC (Sophisticated Interface for Routine Operation with Crystal Centering). After mounting a new sample on the goniometer by the sample exchange robot, SIROCC recognizes a sample loop, and perform two raster scans over the loop regions. From a heatmap based on the number of diffraction spots below $4 \AA$ resolution, SIROCC recognizes a shape and location of a protein crystal and places it to the X-ray beam position. By taking two snapshots, SIROCC evaluates the diffraction quality of the crystal, and if it exceeds a user's defined threshold, SIROCC collects a complete diffraction data set. In a fully automated data collection beamtime, a beamline staff loads samples from users, and starts the automated data collection. Then all samples are mounted and diffraction measurements by SIROCC are performed in a fully automated manner. For a remote interactive data collection, NoMachine Workstation is installed on a workstation running the control software at each beamline. Furthermore, NoMachine Cloud Server, which federates the workstations at all beamlines, is installed on a gateway server which is accessible from outside the facility. At the beginning of a remote access beamtime, a beamline operating staff prepares the beamline, and give a permission for a user to access to the beamline through the gateway server. Then, the user connects through a NoMachine remote desktop software, allowing users to perform measurements remotely from outside the facility. All experimental information from the fully automated or remote data collection are recorded in the database system, PReMo [2]. PReMo also functions as a data processing and analysis pipeline, and users can obtain the experimental information and the result of data processing and analysis on the Web. Recently, a dedicated server for data download is opened, and users can download a diffraction data or data processing result to perform a further analysis with their own workstation immediately after the data collection.

In remote access or fully automated data collection, the user packs frozen samples in Uni-pucks and ships them to the PF using a dry sipper. The beamline staff receives the dry sipper and transfers to the beamline which the sample is assigned. The Uni-pucks are then taken out from the dry sipper and placed in the liquid nitrogen Dewar of the sample exchange robot. To prevent miss-match of the sample mount on the diffractometer in a fully automated measurement or/and remote experiment, the transportation of the dry-shipper and placing the Uni-puck must be performed without errors. Therefore, it is very important to establish a sample tracking system for the reliable operation of fully automated measurements and remote experiments. Recently, we developed a sample tracking system for those experiments. In this system, the user must attach QR code labels to items such as a dry shipper, hard disk drive and so on to be sent, and place a pin with a two-dimensional barcode into No. 16 of the Uni-puck. When the beamline staff takes any action on the shipped items, the QR code is always read, and the status of the item is updated. The status is stored in a database system and shared among beamline staff members, which helps to prevent miscommunication. The two-dimensional barcode on the pin on No. 16 is read by the sample exchange robot after the Uni-pucks are placed in the Dewar, and the robot recognizes automatically which Uni-puck is set in which position in the Dewar.

In 2020, due to the pandemic situation of COVID-19, approximately $75 \%$ of the beamtime in MX beamlines were used for remote experiments or/and fully automated measurements with users unattended.

[1] Hiraki, M., Yamada, Y., Chavas, L.M., Matsugaki, N., Igarashi, N. \& Wakatsuki, S. (2013). J. Phys.: Conf. Ser. $425,012014$.

[2] Yamada, Y., Matsugaki, N., Chavas, L.M., Hiraki, M., Igarashi, N. \& Wakatsuki, S. (2013). J. Phys.: Conf. Ser. $425,012017$.

\section{Keywords: Synchrotron, automation, remote access}

This research was supported by Platform Project for Supporting Drug Discovery and Life Science Research (Basis for Supporting Innovative Drug Discovery and Life Science Research (BINDS)) from AMED. 